\title{
SEMICLASSICAL CALCULATION OF THE IMAGINARY PART OF THE ION-ION OPTICAL POTENTIAL
}

\author{
A.H. BLIN, M. BRACK, B. HILLER and E. WERNER \\ Institut für Theoretische Physik, Universităt Regensburg, \\ D-8400 Regensburg, F.R.G.
}

Abstract- We use Feshbach's projection formalism to write an equation for the wave function that describes the relative distance between the centers of mass of the two ions. We assume that the Q-space can be truncated at the excitation of $1 p-1 \mathrm{~h}$ in either one or the other ion, leaving one of them in its ground state. In the propagator of the effective potential, the two-body interaction is replaced by the ionion mean field VMF. The matrix elements of the elementary interaction are of finite range, represented by an effective wigner force. The total many-body wave function is antisymmetrized only in the partial intrinsic wave function of each ion. Calculations are done in the Thomas Fermi approximation. The Coulomb energy is not considered.

The model - Using these prescriptions we derived in (1) the imaginary part of the optical potential for heavy ion scattering. In coordinate space it is

$$
\begin{aligned}
W_{R_{1} R^{\prime}}=\int_{d t} e^{i E t} & \left\langle\vec{R}\left|\rho_{M} e^{-i\left(T+V_{M F}\right) t}\right| \vec{R}^{\prime}\right\rangle \iiint \int_{d \vec{r}_{1}} d \vec{r}_{1}^{\prime} d \vec{r}_{4} d \vec{r}_{4}^{\prime}\left\langle\vec{r}_{1}\left|\rho e^{i H_{c} t}\right| \vec{r}_{1}^{\prime}\right\rangle . \\
& \left\langle\vec{r}_{4}\left|\rho e^{-i H_{c} t}\right| \vec{r}_{4}^{\prime}\right\rangle F\left(\vec{R}^{\prime}, \vec{r}_{1}^{\prime}, \vec{r}_{4}^{\prime}\right) F\left(\vec{R}_{1}, \vec{r}_{1}, \vec{r}_{4}\right)
\end{aligned}
$$

with

$$
F\left(\vec{R}, \vec{r}_{1}, \vec{r}_{4}\right)=\iint d \vec{s}_{2} d \vec{s}_{3} \sum_{l} \varphi_{l}^{*}\left(\vec{S}_{2}\right) \varphi_{l}\left(\vec{S}_{3}\right) \vee\left(\vec{R}, \vec{r}_{1}, \vec{r}_{4}, \vec{s}_{2}, \vec{s}_{3}\right)
$$

The quantities $\rho, \bar{\rho}$ denote the hole and particle densities respectively, $\rho_{M}$ is the density associated with the wave function of relative motion. The operators $T$ and $V_{M F}$ are the kinetic energy of relative motion and the ion-ion mean field respectively, $H_{C}$ denotes the intrinsic Hamiltonian of either ion $A$ or $B$. In eq.(1.2) the $\varphi_{\mathcal{Q}}$ denote single particle wave functions. In analogy to (2) we take the twobody interaction $v$ to be an effective wigner force, of finite range $v\left(\vec{R}, \vec{r}_{1}, \vec{r}_{4}, \vec{s}_{2}, \vec{s}_{3}\right)=$ $\delta\left(\vec{s}_{2}-\vec{s}_{3}\right) \delta\left(\vec{r}_{1}-\vec{r}_{4}\right) u\left(\vec{R}+\vec{r}_{1}-\vec{r}_{2}\right)$. This sets the excitations of $1 p$ and 1 h at the same place in one of the ions, say $A$, as well as the virtual excitation in the other ion, but is nonlocal in these two. Then the quantity $F$ simply reduces to the averaged interaction $u$ in the density distribution of the ion which remains in the ground state

$F\left(\vec{R}, \vec{r}_{1}\right)=\int d \vec{s}_{2} \rho\left(\vec{s}_{2}\right) u_{2}\left(\vec{R}+\vec{r}_{1}-\vec{s}_{2}\right)$

We take $u=-u_{0} e^{-\left(r / r_{0}\right)^{2}} ; u_{0}=26.5 \mathrm{MeV}, r_{0}=2.25 \mathrm{fm}$. 
To evaluate $W_{R, R}$ we use the semiclassical approximation (2)

$$
\begin{aligned}
& \left\langle\vec{X}+\frac{\vec{x}}{2}\left|\rho e^{i H_{c} t}\right| \vec{X}-\frac{\vec{x}}{2}\right\rangle=\int_{-\infty}^{\infty} \frac{d \vec{p}}{(2 \pi)^{3}} e^{i \vec{p} \cdot \vec{x}} e^{i t\left(\frac{p^{2}}{2 m}+V_{0}(\vec{X})\right)} \Theta\left(\lambda-\frac{p^{2}}{2 m}-V_{0}(\vec{X})\right)_{(1.5)} \\
& \left\langle\vec{R}\left|\rho_{M} e^{-i\left(T+V_{M F}\right) t}\right| \vec{R}^{\prime}\right\rangle=\int_{-\infty}^{\infty} \frac{d \vec{p}}{(2 \pi)^{3}} e^{-i \vec{p} \cdot \vec{y}} e^{-i t\left(\frac{p^{2}}{2 \mu}+V_{M F}(\vec{Y})\right)}
\end{aligned}
$$

where $m$ and $\mu$ denote the nucleon mass and the reduced mass of the $A$ B system, respectively, and $V_{0}$ is the intrinsic potential of one ion. Following similar manipulations as in (2) one obtains the imaginary part of the optical potential in Wigner space as

$$
\begin{aligned}
W(E, \vec{Y}, \vec{Q})=\frac{1}{(2 \pi)^{5}}\left(\frac{\pi r_{0}^{2}}{\sqrt{2}}\right)^{3 / 2} \int d \vec{X} \int d \overrightarrow{p_{0}} \int d \vec{p} \int d \vec{p}^{\prime} \delta\left(\vec{Q}-\vec{p}_{0}+\vec{p}-\vec{p}\right) \delta\left(E-\frac{p_{0}^{2}}{2 \mu}-\frac{p^{\prime 2}}{2 m}+\frac{p^{2}}{2 m}-V_{M F}(\vec{Y})\right) \\
\cdot\left(\Theta-\frac{p^{2}}{2 m}-V_{0}(\vec{X})\right) \Theta\left(\frac{p^{\prime 2}}{2 m}+V_{0}(\vec{X})-\lambda\right) \exp \left(-\frac{r_{0}^{2}}{2}\left(\vec{p}-\vec{p}^{\prime}\right)^{2}\right) \\
\cdot \mathscr{F}\left[\vec{Y}+\vec{X}, \vec{Q}-\vec{p}_{0}\right]
\end{aligned}
$$

$\mathcal{F}\left[\vec{Y}+\vec{X}, \vec{Q}-\vec{p}_{0}\right]=\int d \vec{\xi}^{\prime} e^{-\frac{z}{x_{0}^{2}}\left(\vec{Y}+\vec{X}-\vec{\xi}^{\prime}\right)^{2}} \int d \vec{\xi} e^{i \vec{\xi} \cdot\left(\vec{Q}-\overrightarrow{p_{0}}\right)} \rho\left(\vec{\xi}^{\prime}-\vec{\xi} / 2\right) \rho\left(\vec{\xi}^{\prime}+\vec{\xi} / 2\right)$ The coordinate $\vec{Y}=\frac{\vec{R}+\vec{R}}{2}$ and $\vec{Q}$ is its conjugate momentum. The two delta-functions in (1.7) describe momentum and energy conservation, the $\theta$ functions take the phase space for particles, holes in account.

Calculations - Calculations are performed for a symmetric system. For the ion density profiles we use Gaussians. We use a local $V_{M F}$, parametrized as a Gaussian (1).

The imaginary part $W(E, Y, Q)-A 11$ results are on the energy she11 $E=\frac{Q^{2}}{2}+V_{M A F}$. For Gaussian densities the calculation of $W(E, Y, Q)$ is reduced to a two-fold integration

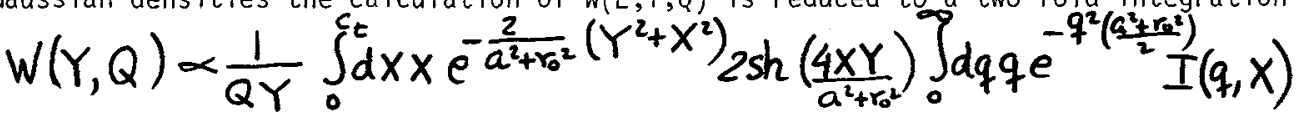
where $c_{t}$ denotes the classical turning point in $V_{0}(X)$ and $I(q, X)$ is a function which contains the integration over all nested ranges of moment and is too lengthy to be shown here.

First we discuss the results for $W$ as function of the incident energy $0<E / \lambda<150$ ( $\lambda$ is the Fermi energy) for a symmetric system $A=64$.

In Fig. 1 we show $W$ calculated with and without the ion-ion mean field $V_{M F}$, for a fixed distance $Y=5.5 \mathrm{fm}$. At his Targe overlap of the densities, the mean field has a strong influence on $H$, specially for smaller incident energies $E / \lambda<50$. Whereas in the absence of $V_{M F}, W$ starts at 0 at $E=0$, and increases sharply, the presence of $V_{M F}$ leads to a slowiy decreasing $W(E)$. The reason is that in the interaction region $V_{\text {uf }}$ is much larger than the incident energy (asymptotoic kinetic energy), i.e. its contribution to the kinetic energy at a given approach distance between the ions is dominant. 


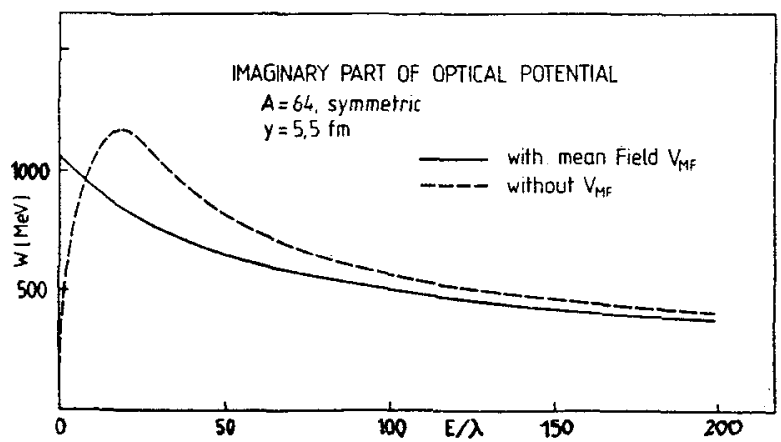

Fig. 1 - Energy dependence of the imaginary part of the optical potential $W(E, Y)$ $(\lambda=47 \mathrm{MeV})$ at fixed distance $Y=5.5 \mathrm{fm}$ between the ions with Gaussian densities. Full line - mean field included; dashed line - no-mean field.

Of course, if the overlap of the densities is small (e.g.Y $\geqslant 8 \mathrm{fm})$, the inclusion of $V_{M F}$ just slightly shifts the energy scale. The spatial dependence of $W$ is shown in fig.2, it is roughily of Gaussian shape for all energies.

Finally in Fig. 3 we show for the $A=12$ system the calculated absorption cross section $\sigma_{a b s}$ for straight 1 ine trajectories in comparison to the experimental ${ }^{12} \mathrm{C}$ reaction cross section oreaction (3) and the experimental ${ }^{12} \mathrm{C}$ inelastic scattering to the 4.4. MeV, $2^{+}$state of ${ }^{12} \mathrm{C}, \sigma_{2}{ }^{+}$. Coherent excitations such as the latter are not described in our model. Since they represent only a small fraction of oreaction, it is legitimate to compare our calculation with the reaction cross section. It is satisfactory that the calculated $\sigma_{a b s}$ lies below the experimental oreaction; this leaves roon (about a factor 2-3) for other than 1p-1h excitations.

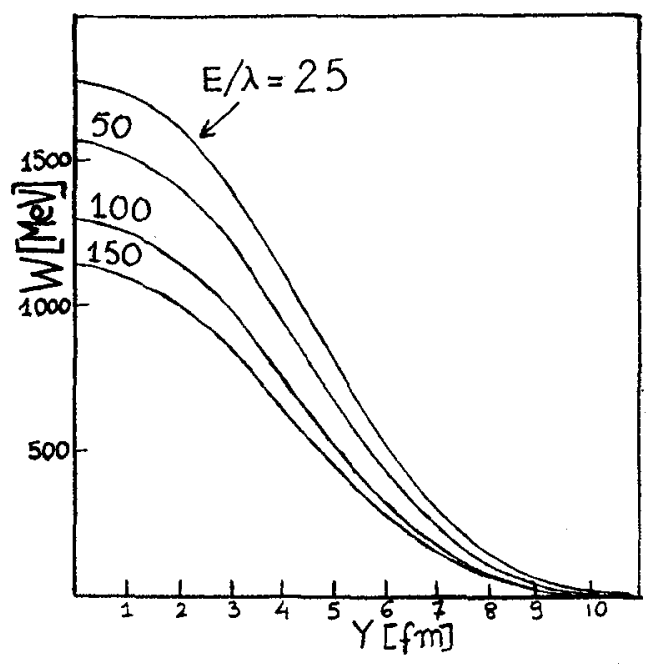

Fig. 2 - Spatial dependence of $W$ for fixed values of the incident energy $E / \lambda$. 


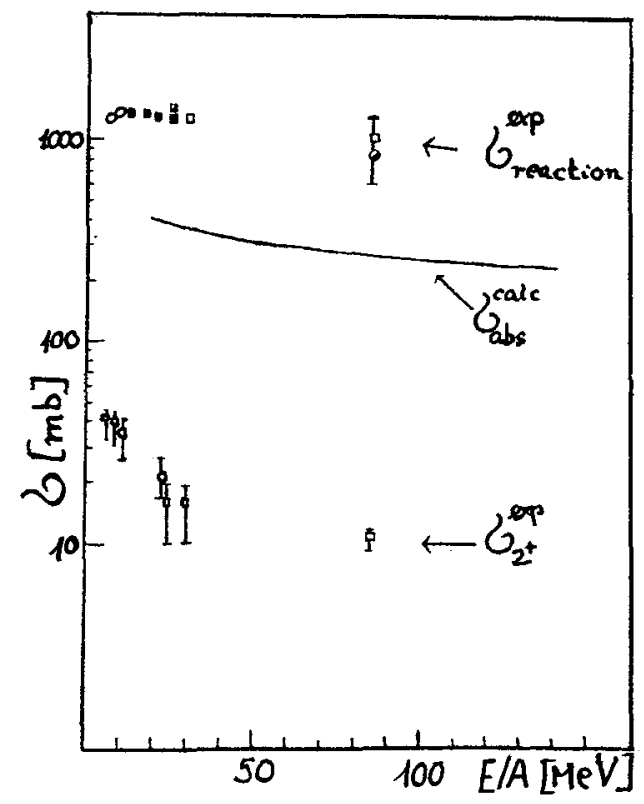

Fig.3 - The calculated absorption cross section for the ${ }^{12} \mathrm{C}+{ }^{12} \mathrm{C}$ reaction compared to the experimental reaction cross section and experimental ${ }^{12} \mathrm{C}$ inelastic scattering to the $2^{+}$ state (3).

\section{Outlook}

We plan to extend this formalism to energies up to $1 \mathrm{GeV} / \mathrm{N}$. To do that the effective two-body interaction has to be adjusted adequately and we have to correct for relativistic effects. At these high energies the neglection of Coulomb distortion and the Hartree approximation become even more realistic.

\section{References}

1 - A.H. BTin, M. Brack, B. Hiller and E. Werner, Proceedings of XXI Winter School on Physics, Part 1, Zakopane, Poland, April 1986;

Proceedings of Int. Workshop on Gross Properties of nuclei and nuclear excitations XV, Hirschegg, Austria, January 1987.

2 - R.W. Hasse and P. Schuck, Nucl.Phys. A438 (1985), 157

3 - M. Buenerd et a1., Nucl.Phys.A424 (1984), 313 